\title{
Resistance profiles and biofilm formation of coagulase negative staphylococci isolated from clinical specimens in a tertiary care hospital in Palestine
}

\author{
Nahed A. Al Laham¹, Emad Abou Elkhair², \\ Abdallah Bashir ${ }^{2}$, Nahed Abdelateef ${ }^{3}$
}

\section{Abstract}

Background: Coagulase-negative staphylococci (CoNS) represent one of the major resistant nosocomial pathogens where its biofilmrelated infections often fail to respond to antibiotic chemotherapy. Here, we studied the resistance profiles and biofilm formation in CoNS isolates from clinical specimens at Al Shifa hospital in Gaza, Palestine.

Methods: This study was carried out from March to July 2013 and included 81 clinical isolates. Identification and antibiotic susceptibility testing were performed using VITEK-2 system. The presence of nuc and mecA genes was performed using multiplex PCR. Qualitative and quantitative biofilm assays were performed using standard methods.

Results: Of the 81 clinical CoNS isolates, S. haemolyticus was the most common species (34, 42\%), followed by S. epidermidis $(26,32.1 \%)$ and S. saprophyticus $(13,16 \%)$. The majority of isolates (83.9\%) were from surgery, ICUs, pediatrics and medicine wards and the most common source was pus $(28,34.6 \%)$. Antibiotic resistance was highest against aminoglycosides, $\beta$-lactams, carbapenems, cephalosporins, fluoroquinolones, fosfomycin and macrolides. Though, no resistance was detected against rifampicin, vancomycin, teicoplanin, nitrofurantoin, linezolid and mupirocin. The antibiotic resistance among MR-CoNS was significantly higher than that among MS-CoNS. Nearly $88.9 \%$ of isolates were multidrug resistant with higher percentage among MRCoNS. Most S. epidermidis (76.9\%) isolates were biofilm producer, with statistically significant association between methicillin resistance and biofilm production.
1 Department of Laboratory Medicine, Faculty of Applied Medical Sciences, Al Azhar University-Gaza, Gaza Strip, Palestine.

2 Department of Biology, Faculty of Science, Al Azhar University-Gaza, Gaza Strip, Palestine.

3 The Central Laboratories, Ministry of Health, Gaza Strip, Palestine.

\section{Contact information:}

Dr. Nahed A. Al Laham.

Address: Department of Laboratory Medicine, Faculty of Applied Medical Sciences, Al Azhar University-Gaza, P. 0. Box 1277, Gaza Strip, Palestine.

$\risingdotseq$ nahedallaham@gmail.com 
Conclusions: High rates of antibiotic resistance were found among CoNS to commonly used antibiotics and the majority were methicillin and multidrug resistance. Most $S$. epidermidis isolates were biofilm producer. These results justified the necessity for national programs and measures to monitor and manage the usage of antibiotics in the Palestinian hospitals and community.

\section{Keywords}

CoNS; Antibiotic Resistance Profile; Methicillin Resistance, Biofilm Formation; Gaza; Palestine.
Received 11-01-2018; Accepted 25-01-2018

\section{Introduction}

Coagulase-negative staphylococci (CoNS) are now representing one of the major nosocomial pathogens and among the most frequently isolated bacteria in the clinical microbiology laboratories [1-5]. They are responsible for bacteremia, endocarditis, mediastinitis, meningitis and progressive joint destruction mainly in patients with neutropenia, indwelling foreign devices, intravascular catheters or other foreign bodies [1, 3, 4]. The clinical most relevant CoNS are Staphylococcus epidermidis, S. lugdunensis, S. saprophyticus, and S. capitis $[6,7]$.

Biofilm production by CoNS, specially S. epidermidis, is considered as an important factor in the pathogenesis of implanted medical devices associated infections [1, 2, 8, 9]. Biofilm formation takes place in four successive phases: the attachment of the bacteria to biotic or abiotic surface; the proliferation and accumulation of bacteria in multilayered cell clusters; the growth of biofilm into a thick and structured layer, and finally the detachment and circulation of single cells or cell agglomerates via the bloodstream [1, 2].

Many literature surveys revealed that CoNS showed high resistance against most of the commonly used therapeutic antibacterial agents including methicillin. Irrespective of geographical locations, a worldwide
SENTRY study showed that, about $70-75 \%$ of CoNS are resistant to methicillin [10]. There is a significant increase in the methicillin-resistant coagulase negative staphylococci (MR-CoNS) infections and these bacteria have recently started to gain resistance to other widely used antibiotics [11-14].

The increased recognition of pathogenic potential of CoNS and emergence of drug resistance among them justify the need to identify various species of CoNS and determine their antibiotic resistance pattern. Epidemiological data about CoNS in Palestine are either scarce or insufficient [15]. To the best of our knowledge, this is the first report describing clinical CoNS infection within the Gaza Strip hospitals. To that end, we conducted this study to determine the frequency of CoNS species isolated from various clinical specimens and to assess their resistance profile to most commonly used antibiotics at Al Shifa hospital in Gaza Strip. Moreover, biofilm forming capacity of $S$. epidermidis isolates was investigated.

\section{Materials and Methods}

\section{Study design and setting}

This study was conducted for five months between March and July 2013 at the clinical microbiology laboratory of the largest medical complex hospital 
in Gaza Strip, Al-Shifa hospital with 500 acute care beds. The study was approved by the department of human resources and development in the ministry of health $(\mathrm{MOH})$ and by the Helsinki committee at the $\mathrm{MOH}$ in Gaza Strip (Approval no. PHRC/ $\mathrm{HC} / 36 / 14)$.

\section{Sampling and bacterial isolates}

Eighty one non-duplicate CoNS isolates associated with diverse clinical infections were collected. The isolates represent different sources including pus, urine, blood, sputum, burn and surgical swabs of hospitalized patients in surgery, pediatrics, medicine, burns, gynecology and ICUs departments. These isolates were collected in 2013, out of a total unique 1121 bacterial isolates.

\section{Isolation and Identification of CoNS and MR-CoNS}

\section{Conventional techniques}

CoNS isolates were identified phenotypically based on colonial morphology, Gram stain, and by using the following methods: catalase test, tube coagulase test, Pastorex ${ }^{\mathrm{TM}}$ Staph Plus latex agglutination (Bio-Rad, Hercules, California), and the Staph ID 32 API system (bioMérieux, France) according to the manufacturer's instructions. VITEK-2 automated system was used to identify CoNS to the species level using VITEK-2 database, version 4.03 (bioMérieux, Marcy l'Etoile, France). Screening for MR-CoNS was done by testing for oxacillinand cefoxitinresistance using the VITEK-2 Compact Gram Positive Card (bio-Mérieux, Paris, France).

\section{Molecular techniques}

DNA isolation: Isolates were overnight subcultured in brain heart infusion broth and pelleted by centrifugation at 5, $000 \mathrm{rpm}$ for $15 \mathrm{~min}$, resuspended in $185 \mu$ of TE buffer (20 mMTris chloride, $2 \mathrm{mM}$ EDTA [PH 8.0]) with $15 \mu \mathrm{l}$ of recombinant lysostaphin (15 mg/ml; Sigma, St. Louis, Mo.) and incubated at $37^{\circ} \mathrm{C}$ for $30 \mathrm{~min}$, then boiled at $95^{\circ} \mathrm{C}$ for 15 minutes. After cooling to room temperature, the suspension was centrifuged at 10,000 rpm for 5 minutes, and the supernatant was used directly as template for PCR reactions [16].

Detection of nuc and mecA genes:A multiplex PCR assay was used for detection of the nuc gene to exclude any coagulase negative $S$. aureus isolate and mecA gene for detection of methicillin-resistance among CoNS isolates [17, 18]. Briefly, primers mecA-1 (5'-GGGATCATAGCGTCATTATTC-3') and mecA-2 (5'-AACGATTGTGACACGATAGCC-3') for the gene mecA and nuc-1 (5'-TCAGCAAATGCATCACAAACAG-3') and nuc-2 (5'-CGTAAATGCACTTGCTTCAGG-3') for the gene nuc were used in a multiplex PCR reaction on a T100' Th Thermal Cycler (BioRad, USA) under the following conditions: an initial 5-min denaturation step at $95^{\circ} \mathrm{C}$, followed by 30 cycles of $1 \mathrm{~min}$ of denaturation at $95^{\circ} \mathrm{C}, 1 \mathrm{~min}$ of annealing at $59^{\circ} \mathrm{C}$, and 2 min of extension at $72^{\circ} \mathrm{C}$; with a final extension step at $72^{\circ} \mathrm{C}$ for $10 \mathrm{~min}$. The sizes of the amplicons were 530bp and 280bp for mecA and nuc genes, respectively.

\section{Antimicrobial susceptibility testing}

Antibiotic susceptibility testing for the CoNS isolates was performed with the automated VITEK-2 Compact automated system, using the VITEK-2 Compact Gram Positive Card (bio- Mérieux, Paris, France); Multidrug-resistant (MDR) phenotype was defined as acquired non-susceptibility to at least one agent in three or more antimicrobial categories used in this study [19].

\section{Biofilm production}

The biofilm forming capacity of only S. epidermidis strains was investigated qualitatively and quantitatively by methods previously described. For qualitative biofilm production, a simple method using growth on Congo red agar (CRG) for detecting biofilm formation was used [20]. With brain heart infusion agar containing Congo red stain at $0.8 \mathrm{~g} /$ liter (BDH Chemicals Ltd., Poole, United Kingdom), 
biofilm-positive strains yield black colonies with a dry crystalline surface appearance, while biofilmnegative strains mostly give a red colonies.

For quantification assay of biofilm-forming capacity, tests similar to those described previously were applied [9, 21, 22]. Briefly, S. epidermidis strains were cultivated overnight in $10 \mathrm{ml}$ of tryptic soy broth (TSB) supplemented with $0.25 \%$ (wt/vol) glucose. Cultures were diluted in TSB supplemented with $0.25 \%(w t / v o l)$ glucose to a final OD578 of 0.1 and $200 \mu \mathrm{l}$ of the cell suspension was used to inoculate sterile, 96-well flat-bottom polystyrene microtiter plates (Greiner bio-one; Cellstar). After cultivation for $24 \mathrm{~h}$ at $37^{\circ} \mathrm{C}$, the wells were emptied and the contents were gently washed three times with $200 \mu \mathrm{L}$ of PBS (pH 7.2) to remove free-floating planktonic bacteria. The plates were air dried for $10 \mathrm{~min}$. , and the remaining surface-adherent cells were stained with $0.1 \%$ safranin (Serva) for $30 \mathrm{~s}$. Absorbance at $490 \mathrm{~nm}$ (A490) was measured with a Micro-ELISA Autoreader (SpectraMAXGeminiXS; Molecular Devices). Wells containing only sterile TSB served as a background control; their average $A 490$ value was subtracted from all experimental readings. S. epidermidis RP62A and S. carnosus TM300 were used as positive and negative controls, respectively. Each assay for each strain was performed in quadruplicate.

\section{Statistical analysis}

The results were tabulated, encoded and statistically analyzed using Statistical Package for Social Sciences (SPSS ${ }^{\circledR}$ ) program version 17 (Chicago, IL, USA). Fisher's exact 2-tailed and Pearson's $\chi^{2}$ tests were used for categorical variables to compare frequencies of CoNS species and MR-CoNS positivity and negativity. Likewise, percentages as of antibiotic resistance profiles of MR-CoNS and MS-CoNS were compared with Pearson's $\chi^{2}$ or Fisher's exact test as appropriate. $P$-value were calculated and $P<0.05$ was considered statistically significant.

\section{Results}

\section{Identification and distribution of CoNS and MR-CoNS species}

Out of a total 1121 bacterial isolates, 81 strains of CoNS that belong to six species were identified. $S$. haemolyticus was the most common species isolated (34, 42\%), followed by S. epidermidis (26, 32.1\%) and S. saprophyticus (13, 16\%) (Table 1). CoNS were

Table 1. Distribution of the 81 CoNS isolates according to gender, ward, type of specimen, species and resistance to methicillin.

\begin{tabular}{|c|c|c|c|}
\hline & No. & $\%$ & $P$-value \\
\hline \multicolumn{4}{|l|}{ Gender } \\
\hline Males & 42 & 51.9 & \multirow{2}{*}{0.47} \\
\hline Females & 39 & 48.1 & \\
\hline \multicolumn{4}{|l|}{ Ward } \\
\hline Surgery & 23 & 28.4 & \multirow{6}{*}{0.86} \\
\hline ICUs & 17 & 21 & \\
\hline Pediatrics & 15 & 18.5 & \\
\hline Medicine & 13 & 16 & \\
\hline Burns & 10 & 12.3 & \\
\hline Gynecology & 3 & 3.7 & \\
\hline \multicolumn{4}{|l|}{ Specimen } \\
\hline Pus & 28 & 34.6 & \multirow{6}{*}{0.15} \\
\hline Swaba & 19 & 23.5 & \\
\hline Urine & 15 & 18.5 & \\
\hline Blood & 12 & 14.8 & \\
\hline Sputum & 3 & 3.7 & \\
\hline Others ${ }^{b}$ & 4 & 4.9 & \\
\hline \multicolumn{4}{|l|}{ Specimen } \\
\hline S. haemolyticus & 34 & 42 & \\
\hline S. epidermidis & 26 & 32.1 & \\
\hline S. saprophyticus & 13 & 16 & \\
\hline S. hominis & 5 & 6.2 & \\
\hline S. warneri & 2 & 2.5 & \\
\hline S. auricularis & 1 & 1.2 & \\
\hline \multicolumn{4}{|l|}{ Specimen } \\
\hline Resistant & 60 & 74.1 & \multirow{2}{*}{$<0.001^{*}$} \\
\hline Susceptible & 21 & 25.9 & \\
\hline Total & 81 & 100 & \\
\hline $\begin{array}{r}\text { ICUs: Intensive Care } \\
\text { and burn swabs. } \\
\text { and }\end{array}$ & $\begin{array}{l}\text { abs inc } \\
\text { ding } c\end{array}$ & $\begin{array}{l}\text { surgica } \\
\text { spinal, p }\end{array}$ & $\begin{array}{l}\text { te infections } \\
\text { ral, synovial } \\
\text { t difference. }\end{array}$ \\
\hline
\end{tabular}


Table 2. Distribution of CoNS species according to the source of specimen and hospital ward.

\begin{tabular}{|c|c|c|c|c|c|c|c|c|c|c|c|c|c|c|}
\hline \multicolumn{15}{|c|}{ Type of specimen } \\
\hline \multirow{2}{*}{ CoNS spp } & \multicolumn{2}{|c|}{ Pus } & \multicolumn{2}{|c|}{ Swaba } & \multicolumn{2}{|c|}{ Urine } & \multicolumn{2}{|c|}{ Blood } & \multicolumn{2}{|c|}{ Sputum } & \multicolumn{2}{|c|}{ Others ${ }^{b}$} & \multicolumn{2}{|c|}{ Total } \\
\hline & No. & $\%$ & No. & $\%$ & No. & $\%$ & No. & $\%$ & No. & $\%$ & No. & $\%$ & No. & $\%$ \\
\hline S. haemolyticus & 14 & 41.2 & 6 & 17.6 & 5 & 14.7 & 4 & 11.8 & 2 & 5.9 & 3 & 8.8 & 34 & 100 \\
\hline S. epidermidis & 9 & 34.6 & 7 & 26.9 & 4 & 15.4 & 6 & 23.1 & 0 & 0 & 0 & 0 & 26 & 100 \\
\hline S. saprophyticus & 1 & 7.7 & 5 & 38.5 & 5 & 38.5 & 2 & 15.4 & 0 & 0 & 0 & 0 & 13 & 100 \\
\hline S. hominis & 2 & 40 & 0 & 0 & 1 & 20 & 0 & 0 & 1 & 20 & 1 & 20 & 5 & 100 \\
\hline S. warneri & 2 & 100 & 0 & 0 & 0 & 0 & 0 & 0 & 0 & 0 & 0 & 0 & 2 & 100 \\
\hline S. auricularis & 0 & 0 & 1 & 100 & 0 & 0 & 0 & 0 & 0 & 0 & 0 & 0 & 1 & 100 \\
\hline \multicolumn{15}{|c|}{ Type of specimen } \\
\hline \multirow{2}{*}{ Hospital ward } & \multicolumn{2}{|c|}{ Surgery } & \multicolumn{2}{|c|}{ ICU } & \multicolumn{2}{|c|}{ Pediatrics } & \multicolumn{2}{|c|}{ Medicine } & \multicolumn{2}{|c|}{ Burns } & \multicolumn{2}{|c|}{ GYN } & \multicolumn{2}{|c|}{ Total } \\
\hline & No. & $\%$ & No. & $\%$ & No. & $\%$ & No. & $\%$ & No. & $\%$ & No. & $\%$ & No. & $\%$ \\
\hline S. haemolyticus & 10 & 29.4 & 6 & 17.6 & 6 & 17.6 & 6 & 17.6 & 5 & 14.7 & 1 & 2.9 & 34 & 100 \\
\hline S. epidermidis & 8 & 30.8 & 7 & 26.9 & 6 & 23.1 & 2 & 7.7 & 3 & 11.5 & 0 & 0 & 26 & 100 \\
\hline S. saprophyticus & 3 & 23.1 & 2 & 15.4 & 3 & 23.1 & 2 & 15.4 & & 7.7 & 2 & 15.4 & 13 & 100 \\
\hline S. hominis & 2 & 40 & 1 & 20 & 0 & 0 & 1 & 20 & 1 & 20 & 0 & 0 & 5 & 100 \\
\hline S. warneri & 0 & 0 & 1 & 50 & 0 & 0 & 1 & 50 & 0 & 0 & 0 & 0 & 2 & 100 \\
\hline S. auricularis & 0 & 0 & 0 & 0 & 0 & 0 & 1 & 100 & 0 & 0 & 0 & 0 & 1 & 100 \\
\hline
\end{tabular}

a: Swabs including surgical site infections and burn swabs. b: Others including cerebrospinal, pleural, synovial and ascetic fluids. *: Statistical significant difference. ICUs: Intensive Care Units. GYN: Gynecology ward.

isolated from 42 (51.9\%) males and 39 (48.1\%) females. The majority of CoNS isolates (83.9\%) were from surgery, ICUs, pediatrics and medicine wards. Of the ten specimen sources, the most common source of CoNS was pus $(28,34.6 \%)$, followed by swabs $(19,23.5 \%)$, urine $(15,18.5 \%)$ and blood $(12,14.8 \%)$. Screening for MR-CoNS revealed that 60 (74.1\%) were MR-CoNS and 21 (25.9\%) were MS-CoNS. There were no statistically significant differences in the distribution of CoNS according to gender, hospital ward and specimen source $(P=$ $0.47,0.86$, and 0.15 respectively). Yet, a statistically significant difference was found between MR-CoNS and MS-CoNS $(P<0.001)$ (Table 1).

As stated in Table 2, the highest numbers of $S$. haemolyticus and S. epidermidis strains were recovered from pus specimens $(41.2 \%, 34.6 \%$ respectively), while $S$. saprophyticus was mainly recovered from urine and swab specimens (38.5\% each). According to the type of hospital ward, the highest numbers of $S$. hominis, $S$. epidermidis and $S$. haemolyticus strains were recovered from surgery ward $(40 \%, 30.8 \%, 29.4 \%$ respectively), whereas $\mathrm{S}$. saprophyticus was mainly recovered from pediatric and surgery wards (23.1\% each) (Table 2). As shown in Table 3, most of S. haemolyticus and S. epidermidis isolates were methicillin resistance (94.1\%, 88.5\% respectively) with a statistical significant difference in comparison to its counterpart

Table 3. Distribution of CoNS species according to methicillin resistance (mecA positive, cefoxitin \& oxacillin resistance.

\begin{tabular}{|l|c|c|c|c|c|}
\hline \multirow{2}{*}{ CoNS species } & \multicolumn{3}{|c|}{ MR-CoNS } & \multicolumn{3}{c|}{ MS-CoNS } & \multicolumn{1}{c|}{ P-value } \\
\cline { 2 - 6 } & No. & $\%$ & No. & $\%$ & \\
\hline S. haemolyticus & 32 & 94.1 & 2 & 5.9 & $<0.0001$ \\
\hline S. epidermidis & 23 & 88.5 & 3 & 11.5 & $<0.0001$ \\
\hline S. saprophyticus & 0 & 0 & 13 & 100 & N.A. \\
\hline S. hominis & 4 & 80 & 1 & 20 & 0.07 \\
\hline S. warneri & 1 & 50 & 1 & 50 & not applicable \\
\hline S. auricularis & 0 & 0 & 1 & 100 & not applicable \\
\hline Total & 60 & 74.1 & 21 & 25.9 & $<0.001$ \\
\hline
\end{tabular}


Vol. 7 No. 3:4 doi: $10.3823 / 814$ methicillin sensitive $(P<0.0001)$. Surprisingly, all $S$. saprophyticus isolates were methicillin sensitive.

\section{Antimicrobial resistance profiles}

The antimicrobial resistance profile of CoNS isolates to the 28 antibiotics tested was shown in Table 4 and Figure 1. Resistance to multiple classes of antimicrobials was observed among CoNS isolates, in- cluding aminoglycosides, $\beta$-lactams, carbapenems, cephalosporins, fluoroquinolones, fosfomycin and macrolides. However, no resistance was detected to any of the following antibiotics: rifampicin, vancomycin, teicoplanin, nitrofurantoin, linezolid and mupirocin. Yet, a very low to low resistance rate was observed to tigecycline, moxifloxacin and clindamycin $(3.7 \%, 8.4 \%, 13.6 \%$ respectively). CoNS stra-

Table 4. Antimicrobial resistance profile of isolated clinical CoNS strains.

\begin{tabular}{|c|c|c|c|c|c|c|c|}
\hline $\begin{array}{l}\text { Antimicrobial } \\
\text { agents }\end{array}$ & $\begin{array}{c}\text { S. } \\
\text { epidermidis }\end{array}$ & $\begin{array}{c}\text { S. } \\
\text { saprophyticus }\end{array}$ & $\begin{array}{c}\text { S. } \\
\text { hominis }\end{array}$ & $\begin{array}{c}\text { S. } \\
\text { warneri }\end{array}$ & $\begin{array}{c}\text { S. } \\
\text { auricularis }\end{array}$ & $\begin{array}{c}\text { Total } \\
\text { resistance }\end{array}$ & P-value \\
\hline$P$ & 100 & 100 & 100 & 100 & 100 & 98.8 & 1.00 \\
\hline AMP & 100 & 100 & 100 & 100 & 100 & 98.8 & 1.00 \\
\hline SAM & 84.6 & 23.1 & 80 & 50 & 0 & 77.8 & $<0.001$ \\
\hline AMC & 84.6 & 23.1 & 80 & 50 & 0 & 77.8 & $<0.001$ \\
\hline CXM & 84.6 & 23.1 & 80 & 50 & 0 & 77.8 & $<0.001$ \\
\hline CEC & 84.6 & 23.1 & 80 & 50 & 0 & 77.8 & $<0.001$ \\
\hline CTX & 84.6 & 23.1 & 80 & 50 & 0 & 77.8 & $<0.001$ \\
\hline CRO & 84.6 & 23.1 & 80 & 50 & 0 & 77.8 & $<0.001$ \\
\hline IPM & 84.6 & 23.1 & 80 & 50 & 0 & 77.8 & $<0.001$ \\
\hline ERY & 30.8 & 69.2 & 100 & 100 & 100 & 71.6 & $<0.001$ \\
\hline AZM & 30.8 & 69.2 & 100 & 100 & 100 & 71.6 & $<0.001$ \\
\hline CLR & 30.8 & 69.2 & 100 & 100 & 100 & 71.6 & $<0.001$ \\
\hline CLI & 19.2 & 0 & 0 & 0 & 0 & 13.6 & 0.51 \\
\hline TET & 19.2 & 30.8 & 40 & 0 & 0 & 30.9 & 0.08 \\
\hline TGC & 0 & 0 & 0 & 0 & 0 & 3.7 & 0.48 \\
\hline GEN & 34.6 & 0 & 0 & 0 & 0 & 45.7 & $<0.001$ \\
\hline TOB & 42.3 & 0 & 0 & 0 & 0 & 48.1 & $<0.001$ \\
\hline LVX & 53.8 & 0 & 0 & 0 & 0 & 46.9 & $<0.001$ \\
\hline MXF & 7.7 & 0 & 0 & 0 & 0 & 8.4 & 0.63 \\
\hline SXT & 23.1 & 23.1 & 60 & 0 & 0 & 42 & $<0.001$ \\
\hline RIF & 0 & 0 & 0 & 0 & 0 & 0 & - \\
\hline VAN & 0 & 0 & 0 & 0 & 0 & 0 & - \\
\hline TEC & 0 & 0 & 0 & 0 & 0 & 0 & - \\
\hline FOF & 7.7 & 100 & 100 & 100 & 100 & 69.1 & $<0.001$ \\
\hline FUS & 50 & 30.8 & 80 & 0 & 100 & 35.8 & $<0.001$ \\
\hline NIT & 0 & 0 & 0 & 0 & 0 & 0 & - \\
\hline LZD & 0 & 0 & 0 & 0 & 0 & 0 & - \\
\hline MUP & 0 & 0 & 0 & 0 & 0 & 0 & - \\
\hline \multicolumn{8}{|c|}{$\begin{array}{l}\text { *: Values represent percentages P: benzylpenicillin; AMP: ampicillin; SAM: ampicillin/sulbactam; AMC: amoxicillin/clavulanic acid; CXM } \\
\text { cefuroxime sodium; CEC; cefaclor; CTX: cefotaxime; CRO: ceftriaxone; IPM: imipenem; ERY: erythromycin; AZM: azithromycin; CLR } \\
\text { clarithromycin; CLI: clindamycin; TET: tetracycline; GEN: gentamycin; TGC: tigecycline; TOB: tobramycin; LVX: levofloxacin; MXF: moxifloxacin } \\
\text { SXT: trimethoprim-sulfamethoxazole; RIF: rifampin; VAN: vancomycin; TEC: teicoplanin; FOF: fosfomycin; FUS: fusidic acid; NIT: nitrofurantoin } \\
\text { LZD: linezolid; MUP: mupirocin }\end{array}$} \\
\hline
\end{tabular}


Figure 1: Antimicrobial resistance patterns of isolated clinical MR-CoNS and MS-CoNS strains.

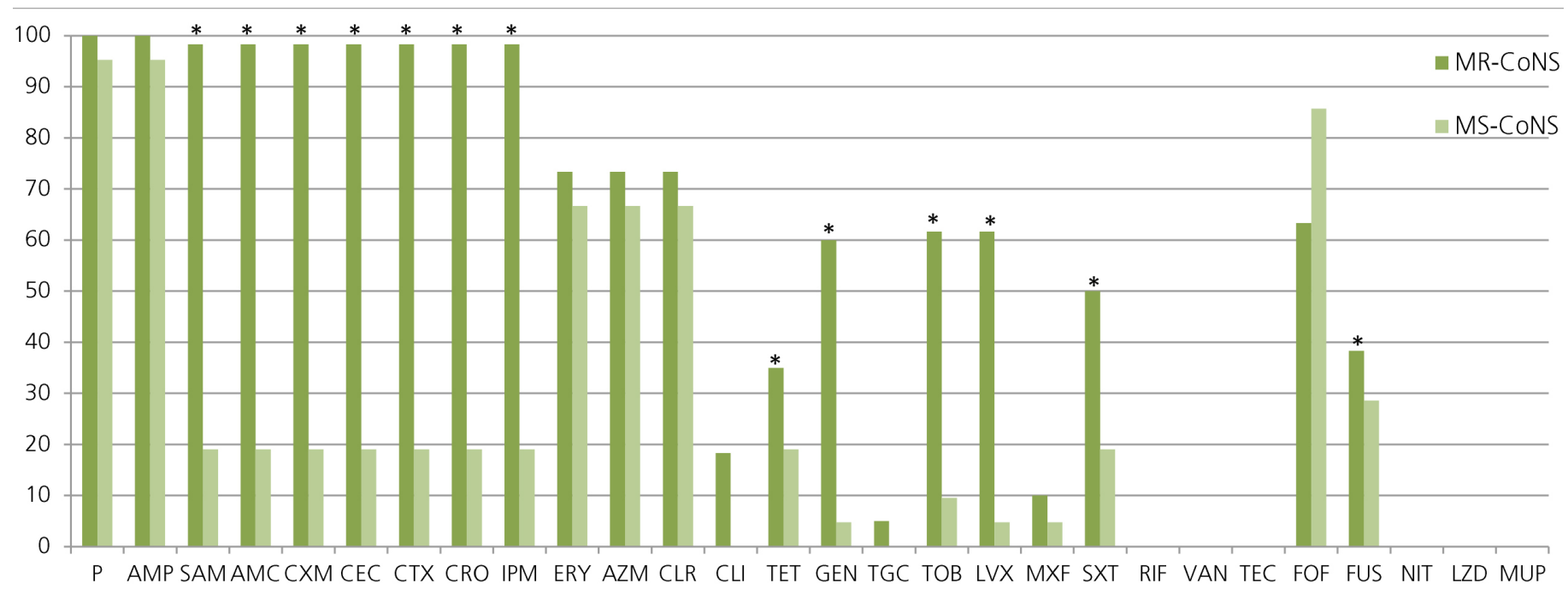

P: benzylpenicillin; AMP: ampicillin; SAM: ampicillin/sulbactam; AMC: amoxicillin/clavulanic acid; CXM: cefuroxime sodium; CEC; cefaclor; CTX: cefotaxime; CRO: ceftriaxone; IPM: imipenem; ERY: erythromycin; AZM: azithromycin; CLR: clarithromycin; CLI: clindamycin; TET: tetracycline; GEN: gentamycin; TGC: tigecycline; TOB: tobramycin; LVX: levofloxacin; MXF: moxifloxacin; SXT: trimethoprim-sulfamethoxazole; RIF: rifampin; VAN: vancomycin; TEC: teicoplanin; FOF: fosfomycin; FUS: fusidic acid; NIT: nitrofurantoin; LZD: linezolid; MUP: mupirocin. *Statistically significant difference in resistance pattern between MR-CoNS and MS-CoNS (P-value $<0.05$ ).

ins exhibited nearly complete resistance to penicillin and ampicillin (98.8\% each). Rates of resistance to other antibiotics were $77.8 \%$ to $\beta$-lactam inhibitors, carbapenems and cephalosporins, $71.6 \%$ to macrolides, $69.1 \%$ to fosfomycin, $48.1 \%$ to tobramycin, $46.9 \%$ to levofloxacin, $45.7 \%$ to gentamicin, $42 \%$ to trimethoprim/sulfamethoxazole (SXT), 35.8\% to fusidic acid, and 30.9\% to tetracycline. S. haemolyticus and $\mathrm{S}$. epidermidis showed a significantly higher resistance rate to a quite number of tested antibiotics in comparison to the other species. Also both species showed low to moderate resistance to clindamycin, tigecycline, gentamycin, tobramycin, levofloxacin and moxifloxacin in comparison to the other CoNS species which were completely susceptible to those aforementioned antibiotics (Table 4).

Resistance to multiple classes of antimicrobials was found among both MR-CoNS and MS-CoNS, including $\beta$-lactams, macrolides, tetracycline, SXT, fosfomycin and fusidic acid. Overall, MR-CoNS strains exhibited complete resistance to penicillin and ampicillin (100\%), and nearly complete resistance to $\beta$-lactam inhibitor antibiotics, cephalosporins and imipenem (98.3\%) (Figure 1). Also, high resistance was observed to macrolides (73.3\%), fosfomycin (63.3\%), tobramycin and levofloxacin (61.7\%), gentamicin (60\%), and moderately to fusidic acid (38.3\%) and tetracycline (35\%). MS-CoNS strains also exhibited nearly complete resistance to penicillin and ampicillin (95.2\%). Resistance rate to fosfomycin, macrolides, fusidic acid, $\beta$-lactam inhibitor antibiotics and cephalosporinswas 85.7\%, 66.7\%, $28.6 \%, 19 \%$ and $19 \%$ respectively. However, none of the MS-CoNS strains were resistant to clindamycin and tigecyclinealong with aforementioned six antibiotics. The resistance rate of MR-CoNS isolates to $\beta$-lactam inhibitor antibiotics, cephalosporins, tetracycline, gentamicin, tobramycin, levofloxacin, $S X T$, and fusidic acid were significantly higher than those of MS-CoNS isolates ( $P$ value $<0.05$ ) (Figure 1). MDR phenotype (defined as acquired nonsusceptibility to at least one agent in three or more antimicrobial categories used in this study) was detected in 71 isolates (88.9\%). 
Vol. 7 No. $3: 4$ doi: $10.3823 / 814$

\section{Biofilm production}

Out of 26 isolates of $\mathbf{S}$. epidermidis examined, a significant number $(20,76.9 \%)$ produced biofilms as verified by formation of black colonies with a metallic sheen after $24 \mathrm{~h}$ of incubation on CRA plates and by polystyrene microtiter plate assay $(P<0.0001) .82 .6 \%$ of which were in MR-CoNS, and $33.3 \%$ in MS-CoNS. Methicillin resistance was found to be significantly higher in biofilm positive strains $(19,82.6 \%)$ than in biofilm negative strains $(4,17.4 \%)(P<0.0001)$ (Table 5). Also, biofilm producer strains were significantly higher among CoNS isolates recovered from pus, swabs and blood specimens $(P=0.0013, P=0.01, P=0.028$ respectively).

Table 5. Distribution of biofilm-producing S. epidermid is according to methicillin resistance and clinical source.

\begin{tabular}{|c|c|c|c|c|c|c|c|}
\hline & \multicolumn{2}{|c|}{$\begin{array}{c}\text { S. } \\
\text { epidermidis }\end{array}$} & \multicolumn{2}{|c|}{$\begin{array}{l}\text { Biofilm } \\
\text { positive }\end{array}$} & \multicolumn{2}{|c|}{$\begin{array}{l}\text { Biofilm } \\
\text { negative }\end{array}$} & \multirow{3}{*}{ P-value } \\
\hline & 26 & & 20 & 76.9 & 6 & 23.1 & \\
\hline & No. & $\%$ & No. & $\%$ & No. & $\%$ & \\
\hline \multicolumn{8}{|c|}{ Methicillin resistance } \\
\hline MR-CONS & 23 & 88.5 & 19 & 82.6 & 4 & 17.4 & $<0.0001$ \\
\hline MS-CoNS & 3 & 11.5 & 1 & 33.3 & 2 & 66.7 & 0.46 \\
\hline \multicolumn{8}{|c|}{ Clinical source } \\
\hline Pus & 9 & 34.6 & 8 & 88.9 & 1 & 11.1 & 0.0013 \\
\hline Swabs & 7 & 26.9 & 6 & 85.7 & 1 & 14.3 & 0.01 \\
\hline Urine & 4 & 15.4 & 1 & 25 & 3 & 75 & 0.19 \\
\hline Blood & 6 & 23.1 & 5 & 83.1 & 1 & 16.7 & 0.028 \\
\hline Total & 26 & 100 & 20 & 76.9 & 6 & 23.1 & 0.0001 \\
\hline
\end{tabular}

\section{Discussion}

Data regarding CoNS and MR-CoNSin Palestine are insufficient, with only one report from Nablus, Palestine [15]. Previous reports from Gaza Strip and West Bank of Palestine have mostly focused on epidemiology of $S$. aureus and MRSA both in hospital and community settings [23-27]. Here, we described for the first time the resistance profiles and biofilm formation in CoNS isolated from clinical specimens in Gaza, Palestine. S. haemolyticus was the most common species isolated (34, 42\%), followed by S. epidermidis (26, 32.1\%). This finding is comparable to the findings in a study conducted in China where the prevalence of $S$. haemolyticus was the highest (34.1\%), followed by S. epidermidis (27.4\%) [4]. Also in another recent study in Brazil, S. haemolyticus was the most prevalent species [9]. However, many studies worldwide showed S. epidermidis is the major isolated species followed by $S$. haemolyticus or other CoNS [10, 11, 28-30]. These differences in CoNS species rates from the worldwide literature are depending on the country, hospital specialty, study design and setting. In this study, the most common clinical source of CoNS was pus (34.6\%), followed by swabs (23.5\%), urine (18.5\%) and blood (14.8\%). Two Indian studies showed that pus specimens were the most common source of CoNS [31, 32]. Furthermore, these results are nearly similar to the study done in Pakistan in which percentage of CoNS isolates in pus was $36.7 \%$, and in swab was $17.3 \%$ but their findings in regard to the blood (45.9\%) was higher [12]. Also, other studies revealed higher CoNS isolates from blood cultures as in Pakistan (53.5\%) [33], Jordan (30.9\%) [11], Tunisia (29\%) [34], and Iran (25.4\%) [35]. Yet, in a recent study from Jordan and in accordance with our findings, the ICU and surgery wards accounted for the majority of all CoNS isolates obtained (41\%) [11]. A plausible explanation for the differences in the isolation rates of CoNS from clinical source and wards between different countries and even some time in the same country may be due in part to differences in sample size or frequency of sampling and using different conventional and/or molecular methods among others for diagnosis. Screening for MR-CoNS revealed that $74.1 \%$ were MR-CoNS which is nearly similar to the studies done at Pakis$\tan (70.3 \%)$ [12], China (70\%) [4], USA (74\%) [36], and India (68.4\%) [37]. Likewise, prevalence of MRCoNS in various parts of Europe is in the range of $60-75 \%$ [38]. On the other hand, there were significant higher rates of MR-CoNS in studies conducted 
in Saudi Arabia and Poland where the prevalence reached $93.6 \%$ and $98 \%$ respectively $[10,39]$. Yet, other studies found less prevalence of MR-CoNS in comparison to our results as from Iran (50\%) [30] and India (32.7\%) [28].

In this study, the resistance profile of all CoNS isolates showed complete to high resistance to most of the antibiotics tested. This pattern of resistance was shown in previous studies in different countries around the world [4, 8, 10-13, 15]. High resistance to the aforementioned antibiotics could be mainly due to excessive use, misuse, and great prescription of these medication in Gaza for both hospital and community acquired infections and also in agriculture and animal feeding. Moreover, the lack of an antibiotic policy and the availability of antibiotics sold without a medical doctor prescription in Palestine worsen the case [27]. In view of the high resistance rates of CoNS to these aforementioned antibiotics, treatment of infections caused by CoNS, particularly S. epidermidis and S. haemolyticus at Al Shifa hospital with these antibiotics may not be effective. In contrast, no resistance of CoNS isolate was detected to rifampicin, vancomycin, teicoplanin, nitrofurantoin, linezolid and mupirocin, and very low resistance was detected to tigecycline and moxifloxacin (3.7\%, $8.4 \%$ respectively). Our findings are in agreement with many studies that showed full activity of most of these aforementioned antimicrobials against clinical CoNS isolates [8, 11, 13, 15].Conversely, many reports from around the world showed different percentage of resistance to vancomycin, teicoplanin and linezolid ranging from $0.8 \%$ to $13 \%[4,12,39]$. We think that this zero resistance in this study to glycopeptides and linezolid may be due to its limited prescription and use in Gaza Strip hospitals. So, the full sensitivity to vancomycin, teicoplanin and linezolid can work perfectly against clinical CoNS isolated in Gaza hospitals as indicated against $S$. aureus in recent reports from Gaza Strip [24, 27]. On the other hand, a very low resistance rate was observed to tigecycline and moxifloxacin $3.7 \%$ and $8.4 \%$ respectively). MR-CoNS showed low to moderate resis- tance ratios against clindamycin (18.3\%), tetracycline (35\%), fusidic acid (38.3\%) and gentamycin (60\%). This picture contradicts a study conducted in Turkey where significantly higher resistance to clindamycin (72\%), tetracycline (60\%) and gentamycin (90\%) was reported. Yet, fusidic acid resistance (25\%) was lower than in our findings [40]. The least resistance species found in this study was S. saprophyticus. Same recent report from Jordan found low resistance rate of $S$. saprophyticus isolated from urine samples [11]. The resistance rate of MR-CoNS isolates to $\beta$-lactam inhibitor antibiotics, cephalosporins, tetracycline, gentamicin, tobramycin, levofloxacin, $S X T$, and fusidic acid were significantly higher than those of MS-CoNS isolates ( $P$ value $<0.05$ ) (Figure 1). This picture is mirrored others elsewhere $[4,8$, $10-13,40]$. MDR phenotype was detected in $88.9 \%$ of CoNS isolates. This high level of MDR in our CoNS collection is similar to many studies conducted in different countries around the world as from China [4], Australia [5], Nigeria [8], Saudi Arabia [10], Jordan [11], Pakistan [12], India [13], West Bank of Palestine [15] and Turkey [40].

With regard to the biofilm production, a significant number of $S$. epidermidis isolates $(20,76.9 \%)$ were producers with significantly higher MR-CoNS $(19,82.6 \%)$ than MS-CoNS strains $(4,17.4 \%)(P<$ 0.0001 ) (Table 5). Koksal et al., found $71 \%$ of $S$. epidermidis strains were biofilm positive with statistically significant association $(P<0.001)$ between MR-CoNS (81\%) and MS-CoNS strains (57\%) [40].

Our study has some limitations. First, it is worthwhile to mention that a number of our isolates could be contaminants and not real pathogens. Second, we performed this study for short time in one referral hospital in Gaza Strip. So, this may underestimate or overestimate the real prevalence of CoNS species and MR-CoNS. Third, molecular typing was not performed, such that the clonality of the CoNS strains cannot be assessed and also the biofilm associated genes were not investigated. 


\section{Conclusion}

Several themes of potential concern are raised by our findings. Most noteworthy is the high prevalence of MR-CoNS and its high resistance profile against most agents tested in this study except for new or not commonly used antibiotics in Gaza Strip. Also, most of $S$. epidermidis isolates were biofilm producers. Furthermore, high numbers of CoNS isolates were shown to be MDR. These results highlight the critical need for monitoring and managing the usage of antibiotics in our hospitals and community.

\section{Acknowledgements}

The corresponding author wants to thank the staff of clinical microbiology laboratory at Al Shifa hospital for their excellent technical assistance. Also, the author would like to thank the German Academic Exchange Service (DAAD) short visit program, Germany and the Erasmus Mundus External Cooperation Window (EM ECW lot III), Belgium, EU for supporting his work during his visits to the University Clinic of Muenster, Germany and the Free University of Brussels, Belgium.

\section{References}

1. von Eiff C, Peters G, Heilmann C. Pathogenesis of infections due to coagulase-negative staphylococci. Lancet Infect Dis 2002; 2:677-85.

2. Becker K, Heilmann C, Peters G. Coagulase-negative staphylococci. Clin Microbiol Rev 2014; 27:870-926.

3. Stuart JI, John MA, Milburn S, Diagre D, Wilson B, Hussain Z. Susceptibility patterns of coagulase-negative staphylococci to several newer antimicrobial agents in comparison with vancomycin and oxacillin. Int J Antimicrob Agents 2011; 37:24852.

4. Ma XX, Wang EH, Liu Y, Luo EJ. Antibiotic susceptibility of coagulase-negative staphylococci (CoNS): emergence of teicoplanin-non-susceptible CoNS strains with inducible resistance to vancomycin. J Med Microbiol 2011; 60:1661-8.

5. Qu Y, Daley AJ, Istivan TS, Garland SM, Deighton MA. Antibiotic susceptibility of coagulase-negative staphylococci isolated from very low birth weight babies: comprehensive comparisons of bacteria at different stages of biofilm formation. Ann Clin Microbiol Antimicrob 2010; 27,9:16.
6. Murray PR. Matrix-assisted laser desorption ionization timeof-flight mass spectrometry: usefulness for taxonomy and epidemiology. Clin Microbiol Infect 2010; 16:1626-1630.

7. Dupont C, Sivadon-Tardy V, Bille E, Dauphin B, Beretti J L, Alvarez AS, et al. Identification of clinical coagulase-negative staphylococci, isolated in microbiology laboratories, by matrix-assisted laser desorption/ionization-time of flight mass spectrometry and two automated systems. Clin Microbiol Infect 2010; 16:998-1004

8. Ayepola OO, Olasupo NA, Egwari LO, Schaumburg F. Antibiotic susceptibility pattern and biofilm formation in coagulase negative staphylococci. J Infect Dev Ctries2014; 15:1643-5.

9. Pedroso SH, Sandes SH, Luiz KC, Dias RS, Filho RA, Serufo JC, et al. Biofilm and toxin profile: A phenotypic and genotypic characterization of coagulase-negative staphylococci isolated from human bloodstream infections. Microb Pathog 2016; 100:312-318.

10. Khan MM, Faiz A, Ashshi AM. Clinically significant Coagulase Negative Staphylococci and their antibiotic resistance pattern in a tertiary care hospital. J Pak Med Assoc 2014; 64:1171-4.

11. Al Tayyar IA, Al-Zoubi MS, Hussein E, Khudairat S, Sarosiekf K. Prevalence and antimicrobial susceptibility pattern of coagulasenegative staphylococci (CoNS) isolated from clinical specimens in Northern of Jordan. Iran J Microbiol 2015; 7:294-301.

12. Ehsan M, Memon Z, Ismail MO, Fatima G. Identification and antibiotic susceptibility pattern of coagulase-negative staphylococci in various clinical specimens. Pak J Med Sci 2013; 29:1420-4

13. Singh S, Dhawan B, Kapil A, Kabra SK, Suri A, Sreenivas V, et al. Coagulase-negative staphylococci causing blood stream infection at an Indian tertiary care hospital: Prevalence, antimicrobial resistance and molecular characterisation. Indian J Med Microbiol 2016; 34:500-505.

14. Dagnew M, Yismaw G, Gizachew M, Gadisa A, Abebe T, Tadesse $T$, et al. Bacterial profile and antimicrobial susceptibility pattern in septicemia suspected patients attending Gondar University Hospital, Northwest Ethiopia. BMC Res Notes 2013; 22,6:283.

15. Almasri M, Abu Hasan N, Sabbah N. Macrolide and lincosamide resistance in staphylococcal clinical isolates in Nablus, Palestine. Turk J Med Sci 2016; 23:1064-70.

16. Mathem B, Mediavilla J, Kreiswirth BN. Sequence analysis of the variable number tandem repeat in Staphylococcus aureus protein A gene: spa typing. Methods MolBiol 2008; 431:285305.

17. Becker K, Roth R, Peters G. Rapid and specific detection of toxigenic Staphylococcus aureus: use of two multiplex PCR enzyme immunoassays for amplification and hybridization of staphylococcal enterotoxin genes, exfoliative toxin genes, and toxic shock syndrome toxin 1 gene. J Clin Microbiol 1998; 36:2548-53.

18. Becker K, Pagnier I, Schuhen B, Wenzelburger F, Friedrich AW, Kipp $F$, et al. Does nasal cocolonization by methicillin-resistant coagulase-negative staphylococci and methicillin-susceptible Staphylococcus aureus strains occur frequently enough to represent a risk of false-positive methicillin-resistant S. aureus determinations by molecular methods? J Clin Microbiol 2006; 44:229-31. 
19. Magiorakos AP, Srinivasan A, Carey RB, Carmeli Y, Falagas ME, Giske CG, et al. Multidrug-resistant, extensively drug-resistant and pandrug-resistant bacteria: an international expert proposal for interim standard definitions for acquired resistance. Clin Microbiol Infect 2012; 18:268-81.

20. Heilmann C, Goetz F. Further characterization of Staphylococcusepidermidis transposon mutants deficient in primary attachment or intercellular adhesion. Zentbl Bakteriol1998; 287:69-83.

21. Mack D, Bartscht K, Fischer C, Rohde H, de Grahl C, Dobinsky $S$, et al. Genetic and biochemical analysis of Staphylococcus epidermidis biofilm accumulation. Methods Enzymol2001; 336:215-39.

22. Al Laham N, Rohde H, Sander G, Fischer A, Hussain M, Heilmann $C$, et al. Augmented expression of polysaccharide intercellular adhesin in a defined Staphylococcus epidermidis mutant with the small-colony-variant phenotype. J Bacteriol 2007; 189:4494501.

23. Adwan K, Jarrar N, Abu-Hijleh A, Adwan G, Awwad E, Salameh Y. Molecular analysis and susceptibility patterns of methicillinresistant Staphylococcus aureus strains causing communityand health care-associated infections in the northern region of Palestine. Am J Infect Control 2013; 41:195-8.

24. Al Laham N, Mediavilla JR, Chen L, Abdelateef N, Elamreen FA, Ginocchio CC, et al. MRSA clonal complex 22 strains harboring toxic shock syndrome toxin (TSST-1) are endemic in the primary hospital in Gaza, Palestine. PLoS One 2015; 10:e0120008.

25. Biber A, Abuelaish I, Rahav G, Raz M, Cohen L, Valinsky L, et al. A typical hospital-acquired methicillin-resistant Staphylococcus aureus clone is widespread in the community in the Gaza strip. PLoS One 2012; 7:e42864.

26. El Aila NA, Al Laham NA, Ayesh BM. Nasal carriage of methicillin resistant Staphylococcus aureus among health care workers at Al Shifa hospital in Gaza Strip. BMC Infect Dis 2017; 5,17:28.

27. Al Laham N. Detection and antibiotic resistance pattern of Staphylococcus aureus and MRSA isolated from healthcare workers nares at Gaza Hospitals, Palestine. IAJAA 2016; doi: $10.3823 / 3779$.

28. Bhatt $P$, Tandel K, Singh A, Mugunthan M, Grover N, Sahni AK. Species distribution and antimicrobial resistance pattern of Coagulase-negative Staphylococci at a tertiary care centre. Med J Armed Forces India 2016; 72:71-4.

29. Asaad AM, AnsarQureshi M, MujeebHasan S. Clinical significance of coagulase-negative staphylococci isolates from nosocomial bloodstream infections. Infect Dis (Lond) 2016; 48:356-60.

30. Talebi M, Shafiee M, Sadeghi J, Moghadam NA, Saifi M, Pourshafie MR. Genotypic Diversity of Methicillin-Resistant Coagulase-Negative Staphylococci Isolated from Inpatients and Outpatients. Microb Drug Resist 2016; 22:147-54.

31. Khadri H, Alzohairy M. Prevalence and antibiotic susceptibility pattern of methicillin-resistant and coagulase-negative staphylococci in a tertiary care hospital in India. Int J Medicine Med Sci2010; 2:116-20.
32. Mohan U, Jindal N, Aggarwal P. Species distribution and antibiotic sensitivity pattern of coagulase negative Staphylococci isolated from various clinical specimens. Indian J Med Microbiol 2002; 20:45-6.

33. Latif M, Usman J, Gilani M, Munir T, Mushtaq M, Anjum R. Coagulase negative staphylococci - a fast emerging threat. J Pak Med Assoc 2015; 65:283-6.

34. Bouchami O, Achour W, Ben Hassen A. Species distribution and antibiotic sensitivity pattern of coagulase-negative Staphylococci other than Staphylococcus epidermidis isolated from various clinical specimens. African J Microbiol Res 2011; 5:1298-1305.

35. Sheikh AF, Mehdinejad M. Identification and determination of coagulase-negative Staphylococci species and antimicrobial susceptibility pattern of isolates from clinical specimens. African J Microbiol Res 2012; 6:1669-1674.

36. Sader HS, Jones RN. Antimicrobial susceptibility of Gram positive bacteria isolated from US medical centers: results of the Daptomycin Surveillance Program (2007-2008). Diagn Microbiol Infect Dis 2009; 65:158-62.

37. Chaudhury A, Kumar AG. In vitro activity of antimicrobial agents against oxacillin resistant staphylococci with special reference to Staphylococcus haemolyticus. Indian J Med Microbiol 2007; 25:50-52.

38. Stefani S, Varaldo PE. Epidemiology of methicillin-resistant staphylococci in Europe. Clin Microbiol Infect 2003; 9:1179-86.

39. Brzychczy-Wloch M, Borszewska-Kornacka M, Gulczynska E, Wojkowska-Mach J, Sulik M, Grzebyk M, et al. Prevalence of antibiotic resistance in multi-drug resistant coagulase-negative staphylococci isolated from invasive infection in very low birth weight neonates in two Polish NICUs. Ann Clin Microbiol Antimicrob 2013; 20,12:41.

40. Koksal F, Yasar H, Samasti M. Antibiotic resistance patterns of coagulase-negative staphylococcus strains isolated from blood cultures of septicemic patients in Turkey. Microbiol Res 2009; 164:404-10.

\section{Publish in The International}

Arabic Journal of Antimicrobial Agents

The Journal is an open access peer-reviewed journal that publishes scientific papers about all aspects of antimicrobials. The journal will publish original research articles, reviews, brief reports and case reports dealing with basic and clinical antibacterial agents, antiviral, antiprotozoals, antituberculuous, antifungal and antihelminthes agents. All manuscripts must be prepared in English, and are subject to a rigorous and fair peer-review process. Accepted papers will immediately appear online. The journal aims to advance the knowledge, attitude and the research of chemotherapy in the Arabic world in cooperation with international, national scientific and public societies as well as research centers with similar aims and objectives. 